\title{
REPARTO DE RECURSOS EN DOS ESPECIES PSAMMÓFILAS DE SAURIOS: \\ Acanthodactylus erythrurus y Psammodromus algirus. ARENAL COSTERO DE ALICANTE
}

\author{
por \\ Eduardo Seva ${ }^{1}$
}

\section{RESUMEN}

Se ha estudiado el reparto de recursos entre dos especies psammófilas abundantes de saurios, Acanthodactylus erythrurus y Psammodromus algirus, que conviven en un área del arenal costero en la provincia de Alicante. El reparto de recursos se ha estimado sobre las tres dimensiones fundamentales del nicho, espacio, alimento y tiempo, a las que se ha añadido la dimensión térmica.

Para dos temporadas distintas, 1980 y 1982, se han hallado diferencias significativas en la alimentación de $A$. erythrurus, que pueden atribuirse a diferencias en la precipitación.

La variación intraspecífica por clases de edad de la amplitud del nicho trófico en $A$. erythrurus es manifiesta, definiéndose la especie como mirmecófaga en las etapas juveniles. En $P$. algirus no se manifiesta tal variación.

La amplitud del nicho temporal horario para los cinco meses comprometidos, difiere claramente de una especie a otra. Tróficamente, $P$. algiurs se comporta en conjunto como la especie más eurifaga.

Ambas especies quedan separadas por el tipo de alimentación y tamaño de presas, fundamentalmente, además del régimen horario de actividad. El resto de las dimensiones (espacio, temperatura cloacal y tiempo estacional) se solapan muy ampliamente.

\section{SUMMARY}

The resource partitioning between two abundant psammophile lizard species, Acanthodactylus erythrurus and Psammodromus algirus, was studied in a coastal sand area of Eastern Spain.

We have studied the resource partitioning on the three fundamental niche dimensions, space, food and time, to wich we have added the thermic dimension.

In two differen seasons, 1980 and 1982, significative trophic differences in $A$. erythrurus are found, and it can atribute to precipitation differences.

The intraspecific variation, by size classes, of the trophic dimension in $A$. erythrurus is apparent, and it is deffined as a myrmecophagic species in juvenile stages. In Ps. algirus there is not such variation.

Temporal niche breadth, by hours, in the five months, differs clearly from one species to another. Trophically, $P_{S}$. algirus behaves like the more euriphagic species.

Both species are fundamentally separated by the food type and food size, further, the horary regime of activity. Other dimensions (space, cloacal temperatures and seasonal time), show very high overlap.

(1) Departamento de Biologia. Facultad de Ciencias. Universidad de Alicante. 


\section{INTRODUCCION}

En muchas ocasiones no sobrepasamos la línea que separa lo imaginativo de lo estrictamente real. A menudo trasladamos el concepto de nicho ecológico de una especie a lo que realmente hace un individuo de la misma, su manera de comportarse, sus necesidades tróficas, temporales, etc.; incluso generalizamos las experiencias realizadas en el laboratorio con pocos ejemplares. Se les asigna sin proponerlo, a los individuos, ciertas cualidades voluntaristas o tan siquiera protagonistas.

Muchas veces se piensa, en base a los datos, construir la secuencia de una jornada cotidiana de un individuo adulto o juvenil, macho o hembra, a imagen de una conducta subjetiva. Y es cierto que cada individuo posee su nicho espacial, trófico y temporal, sus propias pautas de conducta para cada sitio, situación y tiempo, pero también es cierto que existe un compromiso colectivo a nivel de población y de acuerdo a unas normas y condiciones particulares, agrupando factores desde el clima, pendientes, sustratos, etc., variables abióticas, hasta las intrínsecamente relacionadas con la población, como densidad, crecimiento, mortalidad, etc.

A menudo, dos poblaciones de la misma especie, manifiestan un comportamiento completamente distinto cuando, de hecho, solamente les separan unas decenas de kilómetros, diferencias que llegan hasta algo que parece tan constante como las pautas de actividad (Mellado, com. pers. para el mismo tandem de especies, en Almería).

Este fenómeno de disparidad de actuaciones se hace tanto más sobresaliente, cuanto más inestable es el ecosistema con el que se trabaja; en los que las limitaciones imprevistas en la vegetación, pluviosidad, etc., afectan en gran medida al alimento disponible, a los tiempos de actividad y a la ocupación del territorio.

El tema de la actuación de una población en su entorno a través de las tres dimensiones fundamentales del nicho ecológico - espacial, temporal y trófica-, mediante su amplitud, ha ocupado muchas líneas en los últimos años, así como los niveles de competencia en dos o más especies, mediante medidas del solapamiento de sus respectivos nichos.

Esta advertencia preliminar, no es sino una llamada al hecho de que los argumentos y datos suministrados en este trabajo solamente tendrían 
aplicación sobre este territorio, sobre estas dos especies que estudiamos y sobre un plazo de tiempo limitado por el estudio realizado.

Es preciso salir al paso de una generalización en la conducta ecológica de los Lacéridos, y a menudo puede verse que el comportamiento de una especie depende de muchos más factores o variables que las estrictamente estipuladas (espacio, tiempo y alimento), no muy ponderables, eso sí, pero que modulan en conjunto las tres antes citadas dimensiones del nicho, lo suficiente como para que carezcan, por sí solas, de la exclusividad que se les otorgó.

Sin embargo, los datos estaban al alcance después de cinco años de trabajo (Seva y Escarré, 1980; Seva, 1982), y suficientemente abundantes como para ofrecer un panorama general de convivencia de las dos especies más abundantes en el arenal costero: Acanthodactylus erythrurus y Psammodromus algirus. El resto de especies, de Lacértidos, componen un peso muy escaso en la comunidad de arenales, y como se dará cuenta en el desarrollo del trabajo, son fácilmente excluibles del panorama de competencia interespecíficas.

\section{METODOLOGIA}

El presente trabajo es continuación o complemento de otros anteriores (Seva y Escarré, op. cit. Seva y Escarré, 1982; Seva, Ferrándis y Escarré, 1982) sobre un estudio de la comunidad de Lacértidos en un arenal costero de la provincia de Alicante.

$\mathrm{El}$ área en concreto, posee alrededor de $100 \mathrm{Ha}$. recogidas entre dos urbanizaciones y en esencia, tiene bajo nivel de acción antropógena. La vegetación corresponde a la Clase Ammophiletaea, aunque se recogen los diversos estadios de una sucesión ecológica, que se manifiesta por un aumento de la materia orgánica contenida en el suelo, mayor cobertura de la vegetación, y que se traduce en distintos tipos de vegetación que nosotros hemos tipificado en una cuadrícula de estudio de $8.000 \mathrm{~m}^{2}$, en cuatro principales (ver Seva y Escarré, op. cit.).

El área ha sido muestreada durante el período comprendido entre los años 1976 y 1981. En 1982 se realizó una primera campaña dirigida únicamente para recoger datos de alimentación de las especies. Estas son las si- 
guientes: A. erythrurus, Ps. algirus, Ps. hispanicus y Lacerta lepida, pertenecientes a la Familia Lacertidae; Tarentola mauritanica, un geckonido y Chalcides bedriagai, un escíncido. Dentro de la parcela de estudio, y en general, las dos primeras especies son las más abundantes, con una proporción aproximada de 5:1. Ps. hispanicus mantiene una relación con $A$. erythrurus de 1:12, la misma que mantiene L. lepida; Chalcides bedriagai y $T$. mauritanica se presentan solamente a título anecdótico y únicamente han sido capturados cuatro ejemplares en total, a lo largo del tiempo de estudio.

Para el estudio de la distribución espacial, se han capturado los ejemplares, situándolos en superficies de $12.5 \mathrm{~m}^{2}$ dentro de la parcela y el marcado se efectuó por combinación de dedos cortados. Este control permitió posteriormente estudios de censado, crecimiento, migración, reproducción, etc. No se recogieron datos de los ejemplares respecto a la altura de su posición en los arbustos, lo que no ha permitido la evaluación del espacio vertical. la distribución espacial se ha abordado pues, desde el punto de vista de los tipos de vegetación, extraídos por nosotros mediante Análisis Factorial de Correspondencias, y sobre unidades superficiales cuadradas, de $10 \mathrm{~m}$. de lado.

Los datos destinados al estudio del nicho temporal, son extra-cuadrícula. Itinerarios de $1.5 \mathrm{Km}$. de longitud, con frecuencia horaria, se han realizado en uno de los días de cada mes, pudiendo reconocerse (en una amplitud de $5 \mathrm{~m}$.) las dos clases de edad de los individuos, y en la mayoría de las ocasiones para $A$. erythrurus, el sexo de los adultos.

Para el aspecto de la alimentación, se cuenta con fecas que corresponden a la lagartija colirroja de la campaña de 1980 y 1982, y con las de lagartija colilarga de la temprada de 1982. La eficacia de este método de análisis de la alimentación, ya fue discutido en un trabajo anterior (Seva, op. cit.).

La totalidad de los ejemplares, colectados y mantenidos para la recogida de fecas durante tres jornadas en el laboratorio, procedían de los exteriores de la parcela para no introducir modificación alguna en la población objeto de estudio.

Por fin, la temperatura cloacal de los individuos, al igual que la temperatura ambiente, se registró instantáneamente después de la captura, 
mediante un miliamperímetro conectado a un termistor NTC Miniwat, en el que la conversación a la escala térmica era sencilla. Los registros corresponden a las 4-5 horas primeras de actividad, que incluye una gran parte de la actividad total diaria de las especies.

Como medida de la amplitud de los diferentes nichos, se empleó la fórmula de Diversidad $H^{\prime}=-\sum p_{i} \log p_{i}$, de Shanon-Weaver (1963), y utilizada por la mayoría de los autores en este concepto, y donde $\mathrm{p}_{\mathrm{i}}$ representa la proporción o frecuencia de cada categoría considerada. Para homologar diversidades cuando tratamos dimensiones de nicho que no poseen el mismo número de clases, se ha utilizado en todos los casos $\mathrm{H}^{\prime} / \mathrm{H}$ max.

Las medidas de solapamiento de nichos, han tenido dos vías, dependiendo de la idoneidad de los datos. Para valores ajustados a una distribución normal, se utiliza la fórmula empleada por Pianka (1969), derivada de MacArtur y Levins (1967):

$$
\mathrm{S}=\mathrm{e}^{-\left(\left(\mathrm{x}_{1}-\mathrm{x}_{2}\right)^{2 / 2} \mathrm{~d}_{1} \mathrm{~d}_{2}\right)}
$$

donde $\mathrm{x}_{1} \mathrm{y} \mathrm{x}_{2}$ son medias $\mathrm{y} \mathrm{d}_{1} \mathrm{y}_{\mathrm{d}}$ son las derivaciones típicas de ambas distribuciones respectivamente. «S» varía entre 0 y 1 .

En otras ocasiones, y cuando los datos no se ajustan a una distribución normal, se utiliza la fórmula suministrada por Pianka (1973), basada en una fórmula de Levins para valores:

$$
\mathrm{O}=\frac{\sum \mathrm{P}_{\mathrm{ij}} \cdot \mathrm{P}_{\mathrm{ik}}}{\sqrt{\sum \mathrm{P}_{\mathrm{ij}}^{2} \cdot \sum \mathrm{P}_{\mathrm{ik}}{ }}}
$$

donde $\mathrm{P}_{\mathrm{ij}}$ y $\mathrm{P}_{\mathrm{ik}}$ son las proporciones del recurso i que utilizan las especies j y $\mathrm{k}$, respectivamente. Varía igualmente desde 0 hasta 1.

\section{RESULTADOS}

La tabla 1 representa el resumen de los análisis de fecas, correspondientes a los 74 ejemplares de $A$. erythrurus de la temporada de 1982, de 
ORTOPTEROS

HEMIPTEROS

HIMENOPTROS

CERAMBICIDOS

ARACNIDOS

FORMICIDOS

LARVAS

R. COLEOPTEROS

RESTO TOTAL
4

83

49

49

17

301

34

302

9

848
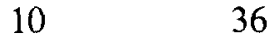

36

12

8

12

16

31

6

51

14

191

Tabla 1. Número de presas por clases, aparecidas en fecas para diferentes temporadas en ambas especies (A.e.: Acanthodactylus erythrurus; Ps. a.: Psammodromus algirus)

los 75 ejemplares de la misma especie para la temporada de 1980 , y de 33 individuos de Ps. algirus de 1982, repartidos entre 9 clases.

Las cifras tan similares de número de fecas de los años 1980 y 1982 en A. erythrurus, nos da pie a la aplicación de un test de comparación de $\mathrm{X}^{2}$ para los meses comunes de muestreo. El valor extraído de $\mathrm{X}^{2}$, homologando el número total de presas aparecidas, es de 54.4 (g.l. $=7, \mathrm{p}<0.001$ ), lo que indica una diferencia notable en el régimnen alimenticio de las dos muestras, aún tratándose de períodos de captura idéntico. En un trabajo anterior (Seva, op. cit.), y comparando análisis de fecas y contenidos del tracto digestivo de diferentes temporadas, tales diferencias tróficas se repitieron, y para cuya explicación se aportaron diferencias significativas en la secuencia y cantidad de precipitación caída durante los meses comprometidos en el análisis. En este caso, dado que las diferencias en las precipitaciones totales son cortas ( $226 \mathrm{~mm}$. en 1982 y $245 \mathrm{~mm}$. en 1980), es necesario buscar las causas en las diferencias de frecuencias registradas en dichos períodos, las que ocasionan el adelanto o el atraso en la aparición de ciertos grupos de presas y que compromete sobre todo a Ortópteros, Coleópteros y Formícidos, principales grupos fuertes en la alimentación de las dos especies (ver Figs. 1 y 2).

Por clases de edad, la alimentación en $A$. erythrurus, se reparte como indica la tabla 2, donde los porcentajes de Ortópteros, Hemípteros y Cerambícidos aumentan con la edad de los animales, y disminuyen en el caso de Formícidos y Larvas. 


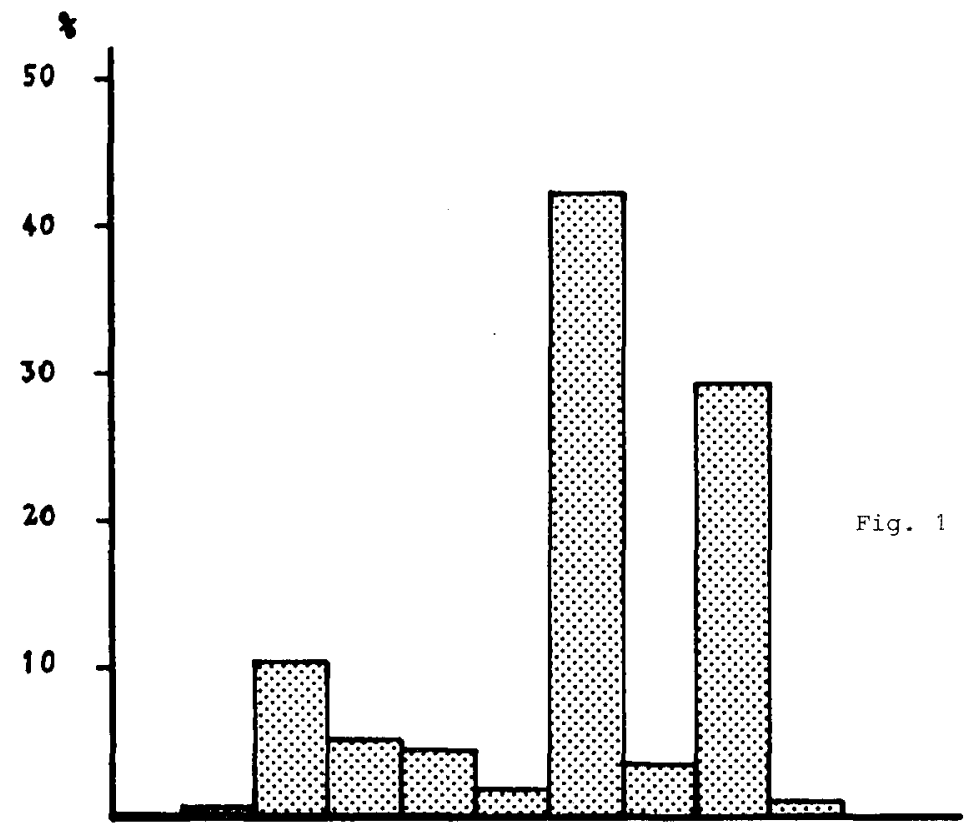

$\begin{array}{lllllllll}1 & 2 & 3 & 4 & 5 & 6 & 7 & 8 & 9\end{array}$

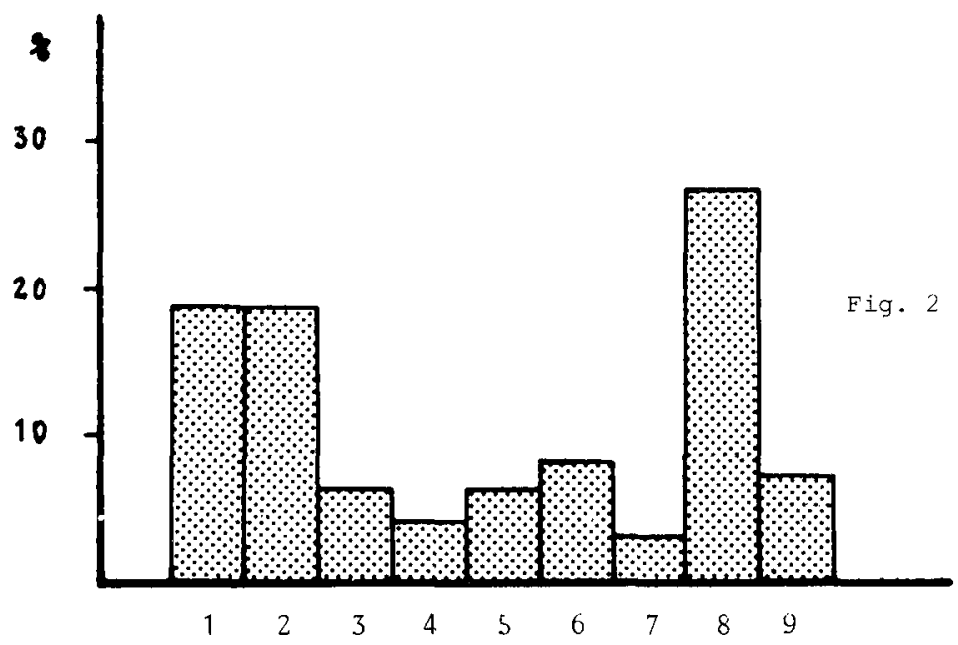

Fig. 1. Histograma de frecunecias de los distintos grupos de presas para la temprada de 1982 Acanthodactylus erythrurus

Fig. 2. Histograma de frecuencias en los distintos grupos de presas para la temprada de 1982 en Psammodromus algirus 
A. erythrurus

Clase edad

(30-42)

(43-52)

(53-62)

(63-76)

ORTOPTEROS
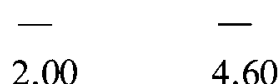

0.08

1.41

HEMIPTEROS

2.00

4.60

12.73

18.36

HIMENOPTEROS

1.00

2.90

7.80

6.49

CERAMBICIDOS

3.30

4.49

8.10

ARACNIDOS

1.00

1.25

2.62

2.54

FORMICIDOS

82.50

43.09

35.58

25.42

LARVAS

5.80

5.99

3.10

R. COLEOPTEROS

12.50

38.49

29.21

32.20

RESTO TOTAL

1.00

0.40

0.70

2.25

PS. algirus

Clase edad

(24-41)

(42-54) (55-72) $\mathrm{mm}$.

ORTOPTEROS

17.64

9.67

25.27

HEMIPTEROS

20.58

11.29

24.17

HIMENOPTEROS

5.88

9.67

4.39

CERAMBICIDOS

3.32

7.70

ARACNIDOS

11.76

8.06

4.39

FORMICIDOS

LARVAS

5.88

16.12

4.39

8.80

1.61

2.20

R. COLEOPTEROS

29.41

24.19

25.27

RESTO TOTAL

16.12

2.20 

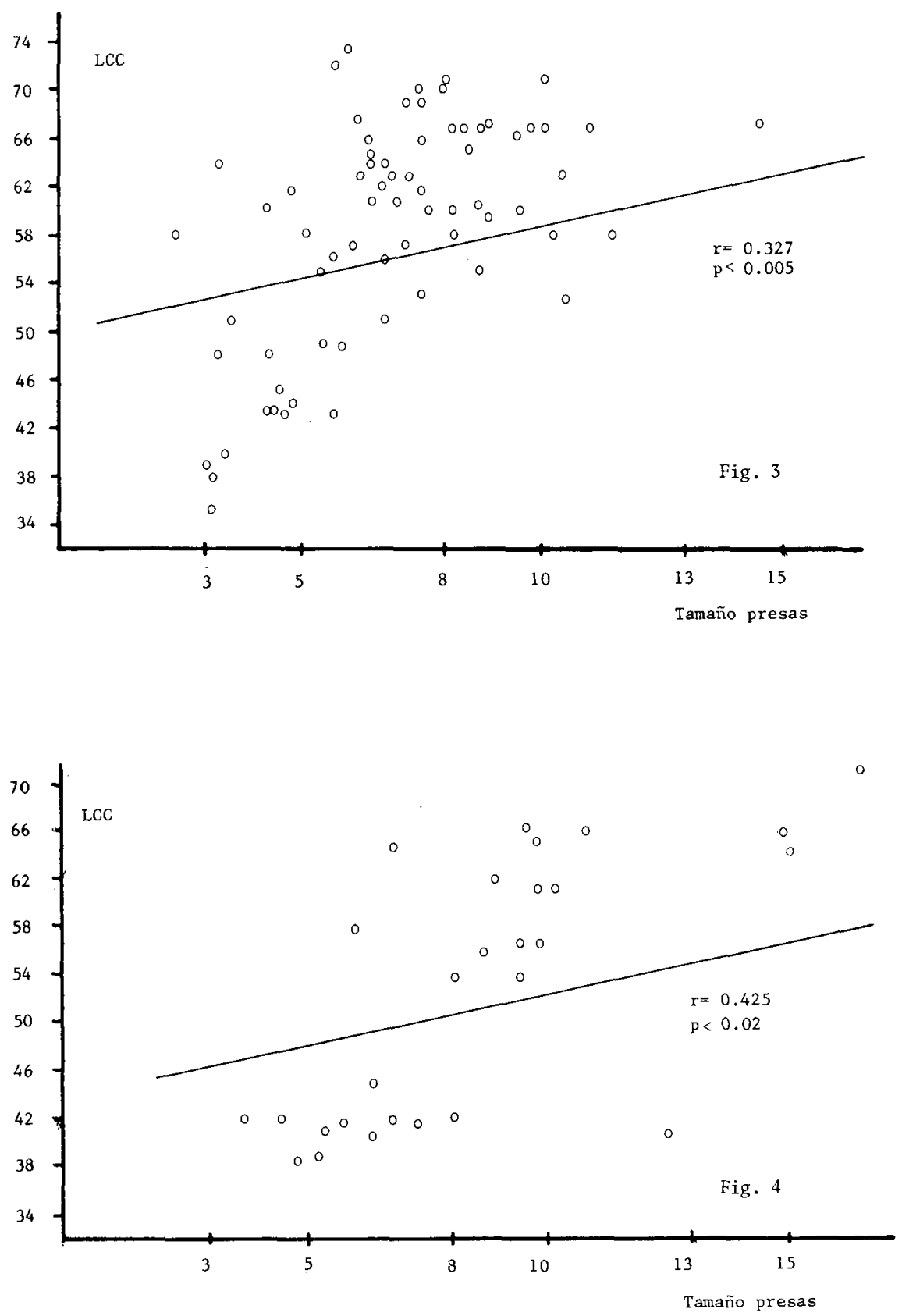

Figs. 3 y 4 . Relación entre tamaños corporales y tamaños de presas para $A$. erythrurus y $P$ s. algirus, respectivamente 
La tabla 3, que muestra los índices de diversidad en la alimentación —dimensión del nicho trófico - por edades, es muy significativa para la lagartija colirroja: índices muy bajos en las clases juveniles, con fuertes presiones sonbre Formícidos y Coleópteros. El hecho de que el índice de diversidad crezca con la edad de los individuos, se debe a la participación de presas de mayor tamaño como Ortópteros y Cerambícidos, fenómeno que se desprende fácilmente de la Fig. 3 en la qụe se relacionan tamaños de individuos y tamaños de presas ingeridas.

Para Ps. algirus, los valores de la dimensión del nicho trófico de la misma tabla 3, se mantienen en un nivel más constante, a la vez que bastante elevado, lo que indica que no ejercen especialidad en la alimentación, ni por clases de edad, ni en conjunto sobre el total de clases de presas, como puede extraerse de la Fig. 2, a excepción hecha -y en comparación a, $A$. erythrurus - de Ortópteros y Formícidos. La relación entre tamaños de los ejemplares y el tamaño medio de las presas, está representado en la Fig. 4.

La tabla 4, representa el número de ejemplares vistos en una jornada de cada uno de los meses del año, para las dos especies. Puede verse que los meses de mayor actividad para ejemplares adultos, corresponde a Abril y Mayo mientras que un segundo pico de actividad en el mes de Agosto, responde fundamentalmente a los nuevos elementos juveniles recién nacidos. Ambas especies mantienen la actividad durante los meses de invierno, representada por muy pocos individuos, y comparativamente mayor en Ps. algirus.

\begin{tabular}{ccccc}
\multicolumn{5}{c}{ Acanthodactylus erythrurus } \\
$\begin{array}{c}\text { Clases edad } \\
\text { (mm.) }\end{array}$ & $(30-42)$ & $(43-52)$ & $(53-62)$ & $(63-76)$ \\
Diversidad & $\mathbf{0 . 2 8 8}$ & $\mathbf{0 . 6 0 4}$ & $\mathbf{0 . 7 5 5}$ & $\mathbf{0 . 7 9 6}$
\end{tabular}

Clases edad

Psammodromus algirus

$\begin{array}{cccc}\text { (mm.) } & (24-41) & (42-54) & (55-72) \\ \text { Diversidad } & \mathbf{0 . 8 1 4} & \mathbf{0 . 9 1 4} & \mathbf{0 . 8 2 5}\end{array}$

Tabla 3. Valores del índice de diversidad para las diferentes clases de edad establecidas en ambas especies 


$\begin{array}{crr}\text { ENERO } & 1 & 1 \\ \text { FEBRERO } & 7 & 4 \\ \text { MARZO } & 62 & 13 \\ \text { ABRIL } & 99 & 27 \\ \text { MAYO } & 113 & 39 \\ \text { JUNIO } & 67 & 11 \\ \text { JULIO } & 56 & 13 \\ \text { AGOSTO } & 111 & 17 \\ \text { SEPTIEMBRE } & 92 & 7 \\ \text { OCTUBRE } & 36 & 7 \\ \text { NOVIEMBRE } & 1 & 4 \\ \text { DICIEMBRE } & 2 & 2\end{array}$

Tabla 4. Número de ejemplares vistos en transecto para cada uno de los meses del año en una jornada, en ambas especies

La ocupación temporal del nicho, dimensionada por los índices de diversidad dados en la tabla 5 , sitúan a las dos especies en una posición semejante en la ocupación estacional - por meses- del sistema. El contaje pormenorizado de individuos a cada hora de la jornada mensual, permite descubrir ciertas diferencias en la dimensión temporal de ambas especies para los cinco meses considerados en la alimentación. La tabla 6 representa los distintos índices de diversidad en la ocupación horaria, y las figuras $5,6,7,8$ y 9 , son la representación gráfica en porcentaje sobre el máximo, de la distribución de los individuos en las diferentes jornadas.

Mientras en $A$. erythurus, las cifras de amplitud del nicho temporal, varían entre márgenes relativamente estrechos (0.76-0.89), en Ps. algirus, estas cifras contienen un rango mayor (0.69-0.91), haciendo uso del ecosistema en fracciones de actuación más numerosas y más cortas cuanto más se aproximan los meses estivales.

Tiempo estac. Espacio Alimento
A. erythrurus
0.839
0.737
0.698
PS. algirus
0.855
0.771
0.896 
En un principio, podría pensarse que las salidas intermitentes de Ps. algirus tendrían que ver con los requerimientos térmicos de esta especie, en la idea de no aceptar temperaturas extremas de mediodía. Pero el registro de temperaturas cloacales de ambas especies, y la búsqueda de la dimensión térmica del nicho de la que habla Pianka (1969, op. cit.), permite conocer que la medida de las temperaturas corporales registradas en 187 individuos, solamente difiere en la primera cifra decimal $\left(33.49^{\circ} \mathrm{C} \mathrm{y}\right.$ $33.13^{\circ} \mathrm{C}$, para $A$. erythrurus y PS. algirus, respectivamente).

Especies que tienen el mismo nicho térmico, tienden a ofrecer el mismo nicho temporal, o dicho de otro modo, la temperatura corporal sería una medida de la dimensión tempral del nicho (Pianka, 1969, op. cit.). Para $A$. erythrurus y Ps. algirus, este presupuesto se cumple para la distribución estacional de ambas, pero no en la ocupación horaria de las distintas jornadas de los meses implicados.

$\begin{array}{lccccc} & \text { Abril } & \text { Mayo } & \text { Junio } & \text { Julio } & \text { Agosto } \\ \text { A. erythrurus } & 0.764 & 0.888 & 0.886 & 0.869 & 0.893 \\ \text { PS. algirus } & 0.875 & 0.910 & 0.768 & 0.693 & 0.761\end{array}$

Tabla 6. Valores de indice de diversidad (amplitud del nicho temporal) en los distintos meses comprometidos para ambas especies

\section{TIPOS DE VEGETACION}

$\begin{array}{lrrrr} & \text { I } & \text { II } & \text { III } & \text { IV } \\ \text { A. erythrurus } & 57 & 107 & 446 & 55 \\ \text { PS. algirus } & 7 & 12 & 56 & 16\end{array}$

Tabla 7. Número de ejemplares para ambas especies capturados en el tiempo de muestreo y clasificados de acuerdo al tipo de vegetación de la parcela de captura

La tabla 7 representa los ejemplares capturados en la parcela de estudio, y clasificados según el tipo de vegetación asignado a cada parcela cuadrada de $10 \mathrm{~m}$. de lado en la que se efectuó la captura. En la misma tabla 5 se indican los índices de amplitud del nicho espacial atendiendo a los tipos de vegetación antes mencionados, considerando que éstos últimos son producto de determinadas características del medio, como la pendiente, tipo de sustrato, grado de humedad, etc. 


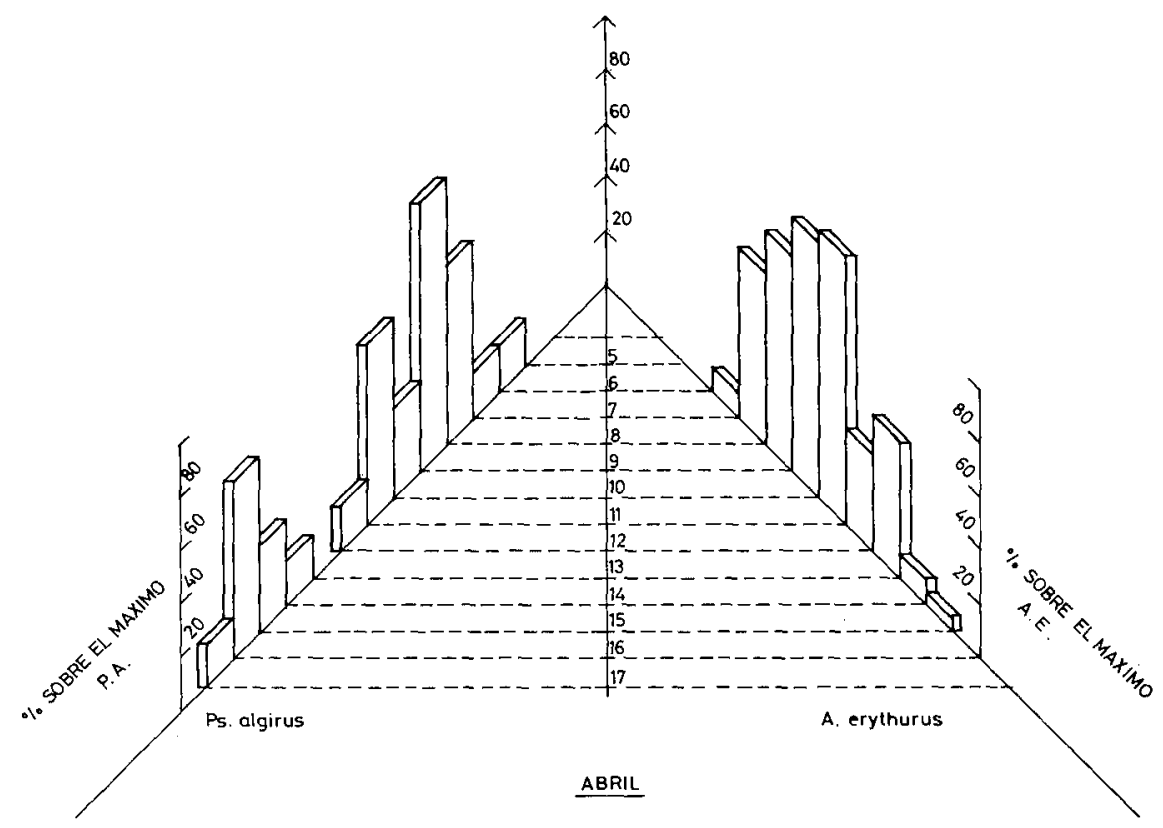

Fig. 5 . Histogramas de actividad diaria para ambas especies

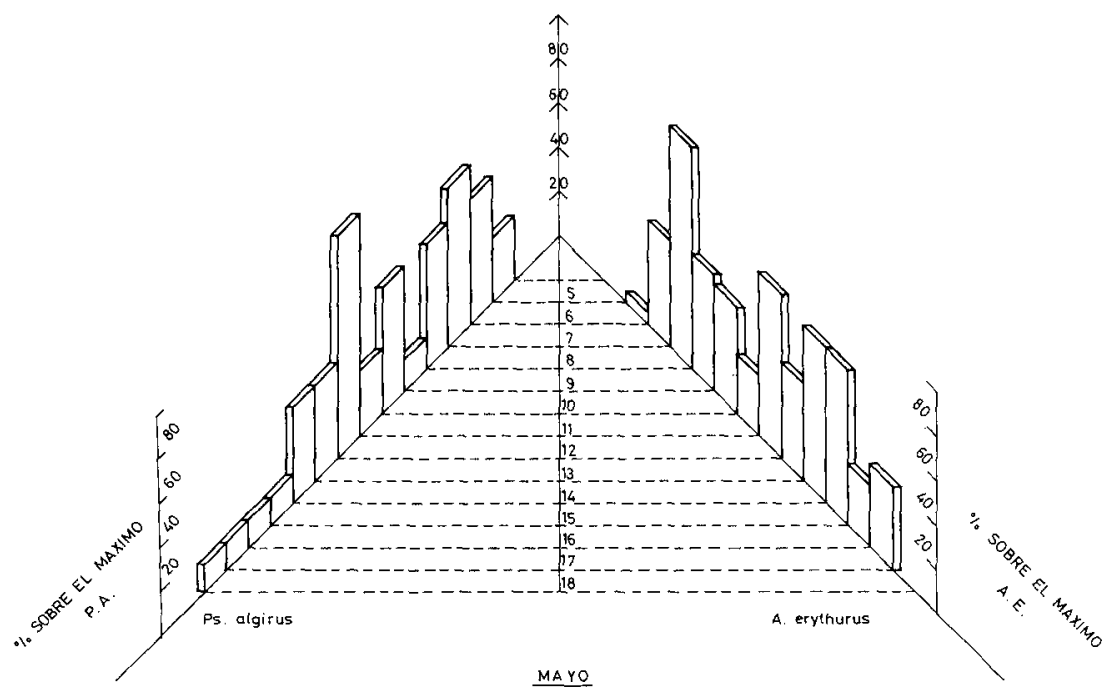

Fig. 6. Hlistogramas de actividad diaria para ambas especies. 


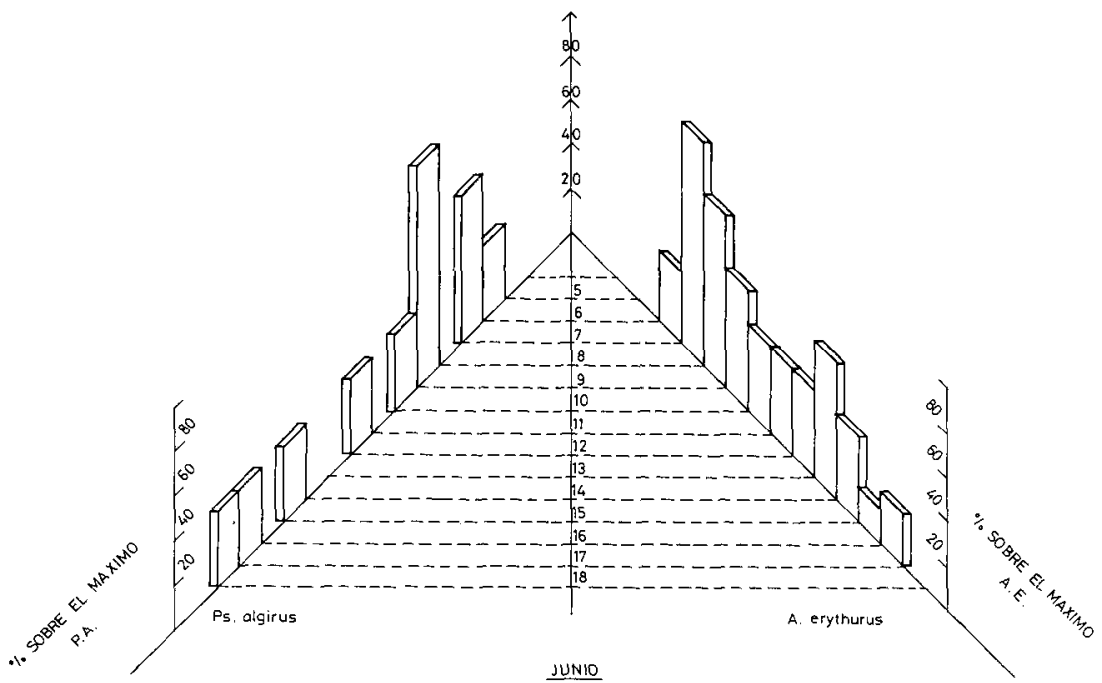

Pig- 7. Listogramas de activiciad diaria para ambas especies.

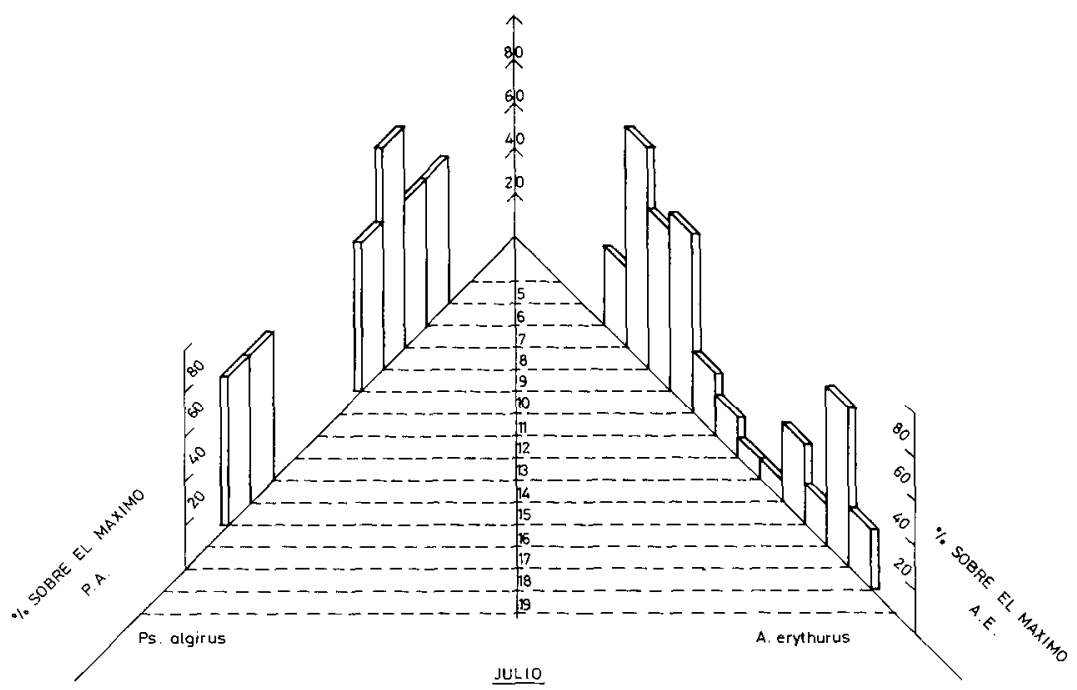

lig. 8. Histogramas de actividad diaria para ambas especics 


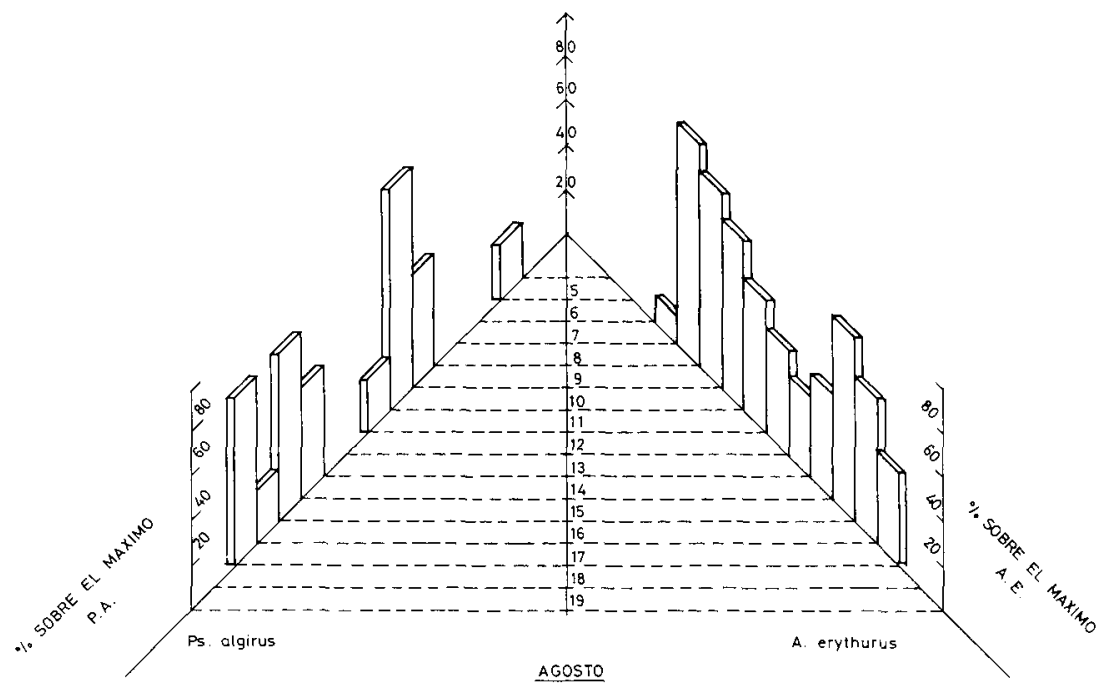

Fig. 9. Histogramas de actividad diaria para ambas especies

Aunque en general, y de acuerdo a la proporción en que se presentan en la parcela los distintos tipos de vegetación, $A$. erythrurus siente preferencia por los tipos III y I, y PS. algirus por los tipos III y IV, las cifras de los índices de diversidad son muy semejantes. Más aún, se efectuó un test de independencia para estas dos especies mediante una tabla de contingencia $2 \times 2$ de presencia-ausencia, en unidades superficiales de $12.5 \mathrm{~m}^{2}$ (Gounot, 1969). Los valores de $\mathrm{X}^{2}$ niegan la independencia en el territorio de las dos especies.

La segregación en el espacio para estos dos saurios, vendría direccionado por el uso de las perchas de los arbustos (Mellado, 1980), para funciones de termorregulación y alimentación. La falta de previsión en la recogida de datos de la correcta posición de los individuos en el primer avistamiento, comprometidos en la caracterización de las observaciones, a1 modo en que lo hacen Mellado y cols. (1975), Mellado (op. cit.) o Schoener (1974), no permiten la cuantificación de esta subdimensión, de gran importancia en el estudio de la ocupación espacial diferencial.

El resto de Lacértidos del arenal, Lacerta lepida y Ps. hispanicus es- 
capan del marco general de competencias de las otras dos. El lagarto ocelado, además de alcanzar toda tipología vegetal en su territorio, su alimentación consta casi exclusivamente de Tenebriónidos de gran tamaño, a los que no tienen acceso normal el resto de los saurios con los que convive. La lagartija cenicienta, hace uso únicamente de las áreas de vegetación tipo I en el espacio, pero su pequeño tamaño y poca abundancia la separan igualmente del marco de las acciones interespecíficas importantes.

\section{DISCUSION}

Acanthodactylus erythrurus y Psammodromus algirus son dos especies de saurios que se hallan en convivencia en la mayoría de los acúmulos arenosos de cierta extensión a lo largo de la costa mediterránea española. La acentuada termofilia de $A$. erythrurus, hace que la proporción entre estas dos especies varíe con la latitud de los enclaves.

En virtud de la escasez de recursos que presentan estas áreas arenosas costeras, la competencia es el mecanismo lógico que media entre especies, como ya sugirió Gause en 1934, cuando poseen similares requerimientos ecológicos y alguno de los recursos escasea.

Las diferencias entre especies, en recurso de alimento, espacio y tiempo, reducen la competencia y permiten la coexistencia de mayor número de especies. Una forma de subrayar la competencia, es la medida del solapamiento de las dimensiones del nicho ecológico, en sus tres fundamentales, espacial, temporal y trófica, a las que nosotros hemos añadido la térmica, como prolongación de la temporal.

En la tabla 8 están representados los índices de solapamiento para las distintas dimensiones del nicho ecológico de ambas especies. En la tabla 9, los índices de solapamiento temporal en los distintos meses que se consideran.

\section{NICHO ESPACIAL}

El solapamiento de los respectivos nichos espaciales en la horizontal, es casi completo (0.988). Aunque la segregación de ciertas zonas con tipos muy concretos de vegetación sea patente por parte de ambas especies, no existen parcelas de exclusión, en las que una de las especies esté ausente, 
existiendo por el contrario, una ocupación simultánea del mismo tipo de recurso espacial.

En el supuesto caso de haber registrado todas las categorías de la situación espacial de los individuos (posición sol-sombra, diversas alturas sobre las plantas, etc.), el solapamiento de los nichos espaciales hubiera sido bastante menor, ya que los ejemplares adultos de $p s$. algirus utilizan en bastantes ocasiones alturas de $10-20 \mathrm{cms}$. sobre arbustos, en la mayoría de las ocasiones, de Crucianella maritima.

El componente espacial parece ser el principal protagonista en la separación entre especies para algunos autores (Mellado y Cols., op. cit.). Y no pienso que sea irreal cuando se trata al menos de dos casos: a, manejo de grandes macrohabitats, y b, manejo de habitats de complicada estructura espacial (espacios multiformes). Cuando incrementamos el número de categorías dentro del espacio por exigencias de su estructura, el distanciamiento entre especies a través de este recurso, será mayor. Como sugiere Heatwole (1977), la estructura física del habitat, puede ser operativa en la selección del habitat. En nuestro caso, aún tratándose de una microdistribución de detalle, las categorías que se pueden distinguir en el espacio son muy reducidas, y dentro de la homogeneidad espacial, los tipos de vegetación parecen una buena criba en las preferencias del espacio.

La competencia por el espacio entre clases de edad de $A$. erythrurus, está suficientemente detallado en un trabajo anterior nuestro (Seva y Escarré, op. cit.).

$\begin{array}{ccccc}\begin{array}{c}\text { Temperatura } \\ \text { corporal }\end{array} & \begin{array}{c}\text { Espacio } \\ \text { (tipos veget.) }\end{array} & \begin{array}{c}\text { Alimento } \\ \text { (Tx. presas) }\end{array} & \begin{array}{c}\text { Alimento } \\ \text { (tmñ. presas) }\end{array} & \begin{array}{c}\text { Tiempo } \\ \text { (estac.) }\end{array} \\ 0.987^{\mathrm{A}} & 0.988^{\mathrm{B}} & 0.659^{\mathrm{B}} & 0.771^{\mathrm{A}} & 0.983^{\mathrm{B}}\end{array}$

Tabla 8. Valores del indice de solapamiento entre $A$ erythrurus y ps. algirus para los distintos recursos que se especifican, utilizando en cada caso los indices $A \circ B$ citados en texto
ABRIL
MAYO
JUNIO
JULIO
AGOSTO
MEDIA
$0.771^{B}$
$0.806^{\mathrm{B}}$
$0.587^{\mathrm{B}}$
$0.628^{\mathrm{B}}$
$0.745^{B}$
0.707 


\section{NICHO TEMPORAL}

Las disputas de protagonismos entre la dimensión espacial y trófica para la separación de especies, resta importancia a la dimensión temporal, y ha sido siempre la que menos atención ha recibido. Las diferencias en los tiempos de actividad, podrían ser un arma efectiva contra la competencia interespecífica si el resto de las dimensiones se hallan solapadas y si los recursos pueden ser renovados con facilidad, como puntualizan MacArthur y Levins (1967).

A. erythrurus y Ps. algirus solapan sus actuaciones en el tiempo de manera casi total (0.983) en la categoría estacional. Aunque el nivel de actividad para meses fríos es ligeramente mayor en la lagartija colilarga (en proporción a su densidad de población), mantiene cotas de actuación muy paralelas a las de la lagartija colirroja, con dos máximos situados en los meses de Mayo y Agosto.

El nicho temporal, en relación con las temperaturas cloacales, puede, en ocasiones, modular el nicho espacial y a su vez, el tipo de alimento que va a encontrar. La temperatura corporal, dice Pianka (1969, op. cit.), es un buen índice de su nicho temporal. Las distribuciones uni o bimodales durante la jornada se relacionan bien con las temperaturas cloacales de los ejemplares. El solapamiento térmico de las dos especies es muy amplio (0.987), semejante al solapamiento temporal estacional; pero existen muy claras divergencias en los periodos de actuación horaria en los distintos meses, y cuyos índices de solapamiento descienden hasta niveles de 0.587 (tabla 9). Esta dosificación temporal de la jornada, no parece que tenga mucha relación con la semejanza térmica de las dos especies, y marcaría la independencia de la dimensión temporal. Este reparto del tiempo diario, reduce competencias en esta dimensión y permite el solapamiento en otras dimensiones cuando los recursos son renovados con facilidad.

\section{NICHO TROFICO}

La dimensión trófica es una de las diferencias más citadas en la separación intra o interespecífica (Schoener, 1977). Dos aspectos importantes es preciso resaltar: 1, las diferencias sustanciales en la alimentación, como ya se ha comentado en el apartado de resultados, en dos temporadas distintas (1980 y 1982) dentro de la misma población de $A$. erythrurus; fenómeno relacionado presumiblemente con los distintos patrones de precipi- 
tación, y que muchos autores siempre han tratado de poner de manifiesto (Mitchell, 1979, recoge gran número de citas a este respecto). 2, las diferencias tróficas entre especies - en taxon de presas y tamaño de las mismas--, y aquellas intraspecíficas por clases de edad en la colirroja.

En la tabla 8 puede verse que las cifras más bajas en el solapamiento de nichos, corresponde a la frecuencia de taxones de presas aparecidas y al tamaño de las mismas. La diferencia en el tipo de alimentación de las dos especies, es debido al distinto planteamiento de estrategias alimentarias. $A$. erythrurus es una especie muy centrada en la mirmecofagia (observar asimismo en Pérez Mellado, 1982 y Valverde, 1967), aunque a la vista de la tabla 2, la clase de adultos saldría de la especialización, haciéndose más oportunista. Ps. algirus observa mayor amplitud trófica (Pérez Mellado, $o p$. cit.), con opciones alimentarias que le brindan las alturas de las perchas por las que cuelga, y una media de tamaño de presas superior a la de $A$. erythrurus, en relación a sus respectivos tamaños corporales.

El solapamiento de nicho trófico intraspecífico entre clases de edad en $A$. erythrurus por taxon de presas, es bajo, pero inferior es todavía el que corresponde a tamaños de presas, por la especialización trófica sobre el grupo Formícidos de las clases juveniles, (tabla 10).

Alimento (n: de
presas por taxa)
0.872
0.537

Tabla 10. Cifras del indice de solapamiento intraspecifico entre las clases de tamaño (30-52) y (53-76) mm. en $A$. erythrurus

Muchas veces se ha argumentado que la participación del territorio proviene de la especialización trófica. En nuestro caso no existe segregación manifiesta en el espacio, y la escasez de recursos del ecosistema aleja los nichos tróficos (tanto intra como interespecíficamente) y dosifica los tiempos de actuación diario de ambas especies.

La cifra de solapamiento global, como producto de los parciales (en el caso del temporal, la media de la de los distintos meses), es en el caso de estas especies, de 0.46 , valor muy próximo al observado para las mismas 
por Pérez Mellado (op. cit.) en el Sistema Central, y para una población de lagartija colirroja realmente reducida. Los valores de solapamiento parcial son, asimismo, muy cercanos a los de Pérez Mellado, lo que da idea de la competitividad de recursos que existe entre estos dos saurios, sin duda por las afinidades de tamaños corporales, adaptaciones al tipo de habitat, etc.

La existencia de sólamente dos especies dominantes en el arenal, se puede interpretar desde el punto de vista de la escasez de recursos (Heatwole, 1976), y de los altos índices de amplitud y solapamiento de nichos en A. erythrurus y PS. algirus.

El estudio de la competencia interespecífica es siempre atractiva, pero no en todo momento los resultados de la labor ortodoxa de campo reflejan la realidad exacta. Los recursos, los nichos, su solapamiento y otros factores que afectan a las poblaciones (densidad y depredación, sobre todo), varían en el tiempo y en el espacio. En palabras de Schoener, los estudiosos de la competencia entre especies son escritores de grandes enciclopedias de un tema, que sólamente han conocido durante unos pocos minutos, y al que se atreven a describior en detalle.

\section{BIBLIOGRAFIA}

GAUSE, G.F. (1934). The Struggle for Existence. 1964 Reprint by N.Y.: Hafner Publ. Co.

GOUNOT, M. (1969). Méthodes d'etude quantitative de la vegetation. Masson. París. 314 pp.

HEATWOLE, H. (1976). Reptile Ecology. University of Queensland Press. Sta. Lucia. Queensland. $178 \mathrm{pp}$.

HEATWOLE, H. (1977). Habitat selection in Reptiles, pp. 137-155 in Gans, C. and D.W. Tinkle Eds. Biology of the Reptilia. Vol 7. Academic Press. London. 720 pp.

McARTHUR, R.H. y LEVINS, R. (1967). The limithing similarity convergence and divergence of coexisting species. Amer. Naturalist 101: 377-385.

MELLADO, J. y cols (1975). The structure of a mediterranean lizard community. Doñana Acta Vertebrata. Vol. II n: 2: 145-160.

MELLADO, J. (1980). Utilización del espacio en una comunidad de Lacértidos del matorral mediterráneo en la Reserva Biológica de Doñana. Doñana Acta Vertebrata 7 (1): 41-49. 
MITCHELL, J.C. (1979). Ecology of southeastern Arizona whiptail lizards (Cnemidophorus: Teiidae): Population densities, resource partitioning and niche overlap. Can. J. Zool. Vol 57: 1487-1499.

PEREZ MELLADO, V. (1982). Estructura de una taxocenosis de Lacertidae (Sauria, reptilia) del Sistema Central. Mediterranea 6: 39-64.

PIANKA, E.R. (1969). Simpatry of desert lizards (Ctenotus) in western Australia. Ecology 50: 1012-1030.

PIANKA, E.R. (1973). The structure of lizards communities. Ann. Rev. Ecol. Syst. 4: 53-74.

SCHOENER, T.W. (1974). Resource partitioning in ecological communities. Science Vol. 185: 27-39.

SCHOENER, T.W. (1977). Competition and the niche. pp. 35-136 in Gans and Tinkle Eds. Biology of the Reptilia. Vol. 7 Academic Press. London. 720 pp.

SEVA, E. (1982). Taxocenosis de Lacértidos en un arenal costero alicantino. Tesis Doctoral. Pub. Univ. Alicante. 317 pp. 10 fot.

SEVA, E. y A. ESCARRE (1980). Distribución espacial y temporal de Acanthodactylus erythrurus (Sauria: Lacertidae) en un arenal costero alicantino. Mediterránea 4: 133-161.

SEVA, E. y A. ESCARRE (1982). Estrategias en el reparto de recursos en cuatro especies de saurios del arenal alicantino. Congreso del Camp d'Alacant. Mayo 1982. Actas Congreso.

SEVA, E. FERRANDIS, E. y A. ESCARRE (1982): La selección del habitat del arenal costero alicantino por $A$. erythrurus (Sauria: Lacertidae). Un proceso markoviano para la identificación de sus tendencias. Mediterránea 6: 5-14.

SHANON, C.E. y W. WEAVER (1963). The mathematical theory of communication. Univ. Illinois. Press. Urbana.

VALVERDE, J.A. 61967). Estructura de una comunidad de Vertebrados Terrestres. Monografías de Ciencia Moderna n. 76. C.S.I.C. Madrid. 220 pp. 